\title{
Structure factor scaling in colloidal phase separation
}

\author{
Juan J. Cerdà, ${ }^{1,2}$ Tomás Sintes, ${ }^{1}$ C. M. Sorensen, ${ }^{2}$ and A. Chakrabarti ${ }^{2}$ \\ ${ }^{1}$ Departament de Física and IMEDEA (CSIC-UIB), Universitat de les Illes Balears \\ and Consejo Superior de Investigaciones Científicas, 07071 Palma de Mallorca, Spain \\ ${ }^{2}$ Department of Physics, Cardwell Hall, Kansas State University, Manhattan, Kansas 66506-2601, USA
}

(Received 26 July 2004; published 19 November 2004)

\begin{abstract}
The dynamical scaling hypothesis for the structure factor, $S(q)$, in depletion-driven colloidal phase separation is studied by carrying out Brownian dynamics simulations. A true dynamical scaling is observed for shallow quenches into the two-phase coexistence region. In such a quench, compact clusters nucleate and grow with time and there is only one characteristic length scale in the system after an initial transient period. Scaling is satisfied beyond this initial period. In contrast, deep quenches lead to fractal cluster growth, and the system is controlled by two characteristic lengths that evolve differently in time [Huang, Oh, and, Sorensen (HOS), Phys. Rev. E 57, 875 (1998)]. True dynamical scaling thus cannot be expected to hold. However, an apparent scaling for the structure factor is observed over some period of time when these two characteristic length scales become comparable to each other. We compare our simulation results for the total structure factor to theoretical predictions by HOS by writing it as a product of cluster-cluster and the averaged single-cluster structure factors, each with its own characteristic length.
\end{abstract}

DOI: 10.1103/PhysRevE.70.051405

PACS number(s): $82.70 . \mathrm{Gg}, 82.70 . \mathrm{Rr}$

\section{INTRODUCTION}

Dynamics of phase separation is the subject of many theoretical and experimental investigations in diverse systems [1-3] such as in binary alloys, liquid mixtures, and polymer blends. Subsequent evolution of the quenched system is determined by the location of the quench inside the phase diagram. In the classical picture, a spinodal line divides the phase diagram into two kinds of instabilities that might govern the dynamical processes: nucleation and spinodal decomposition. If the system is quenched between the spinodal and the coexistence lines (nucleation regime), it becomes unstable against localized, strong amplitude concentration fluctuations. In this situation, nuclei of the minority phase are formed. These nuclei evolve with time in the following way: they grow if their size is larger than a certain critical size, otherwise they dissolve. In the spinodal decomposition regime, the system is unstable against long wavelength, small amplitude concentration fluctuations, which generate an interconnected pattern that coarsens with time. Although this simple picture is of general validity, one can not sharply separate the two regimes and the spinodal line merely serves as a guideline to distinguish which process dominates $[4,5]$.

Late stages of the phase separation process can be described by a dynamical scaling form with a time-dependent characteristic length, $R(t)$. The fundamental assumption of dynamical scaling is that, in late stages of the process, only one length scale is relevant. This characteristic length represents a measure of the typical domain size and increases with time. A major feature of this description is that the pair correlation function $g(r, t)$ and the structure factor $S(q, t)$ depend on time through $R(t)$ only, that is,

$$
g(r, t)=G(r / R(t))
$$

and

$$
S(q, t)=R(t)^{d} F(q R(t)),
$$

where $d$ is the dimensionality of the system and $G$ and $F$ are time-independent universal scaling functions.

Several years ago, small-angle light scattering experiments in moderately dense colloidal solutions [6-9] showed a dynamical scaling behavior surprisingly similar to that observed in binary mixtures undergoing phase separation. In particular, the scattered intensity distribution in a concentrated colloidal solution shows a pronounced peak at a finite value of the wave number, $q_{m}$. Furthermore, the position of the peak moves to smaller values as the aggregation proceeds, while the peak intensity increases. In the later stages of the colloidal aggregation process, the dynamical structure factor, $S(q, t)$, is found to scale according to the following form:

$$
S(q, t)=q_{m}^{-D_{f}} \mathcal{F}\left(q / q_{m}\right),
$$

where $\mathcal{F}(x)$ is a time-independent scaling function, and $D_{f}$ is the fractal dimension of the colloidal clusters. This scaling form is characteristic of the physical systems undergoing phase separation [Eq. (2)], except that, $q_{m}^{-1}$ is considered a characteristic length in the system, and the spatial dimension $d$ of the system is replaced by $D_{f}$, the fractal dimension of the colloidal clusters.

Dynamical scaling of the structure factor in an aggregating colloidal solution is quite unexpected since colloidal aggregation in these experimental systems is irreversible and leads to the formation of fractal aggregates, in contrast to the phase separation processes in binary mixtures. The dynamical evolution of such irreversible colloidal aggregation is well understood in terms of the diffusion-limited-clustercluster aggregation (DLCA) model [10], where the initial colloidal monomers execute a Brownian motion until small clusters are formed, and then the clusters themselves diffuse and aggregate to form even larger clusters. Thus, it is not 
clear why dynamical scaling with only a single length scale will be appropriate for colloidal aggregation. In fact, Huang, Oh, and Sorensen (HOS) [11] have argued that irreversible colloidal aggregation is characterized by two different length scales, namely the mean cluster radius of gyration $R_{g}(t)$ and the mean nearest-neighbor cluster-cluster separation distance $R_{n n}(t)$. Generally speaking, one can write

$$
R_{g}(t) \sim t^{n_{1}} \quad \text { and } \quad R_{n n}(t) \sim t^{n_{2}}
$$

where $n_{1}$ and $n_{2}$ are the respective growth exponents. HOS have demonstrated that these two length scales grow with time with two different temporal exponents $\left(n_{1} \neq n_{2}\right)$ and thus, there is no dynamical scaling of the structure factor in an aggregating colloidal system. This is a direct consequence of the fractal nature of the clusters with a fractal dimension smaller than the space dimension $\left(D_{f}<d\right)$. HOS have further concluded that for monodisperse systems the total structure factor $S(q, t)$ may be written as the product of two different structure factors, each with its own characteristic lengths. The first component, $S_{c c}(q, t)$, is the cluster-cluster contribution to the total structure factor, for which the cluster nearestneighbor separation $R_{n n}(t)$ is the associated characteristic length. The second component, $S_{s c}(q, t)$, is the contribution from particles inside a single cluster with the cluster radius of gyration $R_{g}$ as its characteristic length. HOS have claimed that when clusters are compact, as in the case of phase separation in a binary mixture, both characteristic lengths evolve with the same temporal exponent $\left(n_{1}=n_{2}\right)$. Thus, there would be a single, effective length in the system and dynamical scaling will be satisfied. But when clusters are fractals, dynamical scaling might be satisfied at most over a limited window of time when $R_{g}(t) \approx R_{n n}(t)$, but scaling as a general principle must break down.

Recent experimental and theoretical efforts show that colloidal aggregation can be made reversible by tailoring the strength and range of interaction between colloidal particles. As a result, colloidal solutions can display a rich series of phase transitions between gas, liquid and solid phases $[12,13]$. Manipulation of the interaction potential between colloidal particles can be achieved in several ways. For a charge stabilized colloidal solution, this can be done by the addition of salt or surfactant solution of predetermined molarities so that a secondary minimum [14] in the interaction potential forms. Another way to control the interaction potential between colloidal particles is to induce a depletion interaction $[15,16]$ by adding a nonadsorbing polymer [17] (or a different sized colloid [18]) in an otherwise stable colloidal solution. A major advantage of the latter systems is that the strength and range of the depletion interaction can be easily controlled by varying the polymer concentration and the length of the added polymer chains.

In a recent Brownian dynamics simulation [19] of a twodimensional model of depletion-driven colloids with Asakura-Oosawa (AO) potential, we have shown that a transition from dispersed-phase to a coexistence of dispersedphase and solid-phase takes place as one increases the depth of the depletion potential well. Near the transition point, formation of clusters with a round shape is observed. As the well depth is increased further, one first obtains elongated clusters and then fractal clusters (with fractal dimension $D_{f}$ $\simeq 1.4$ ) form for deep enough well depths. Our simulations also show how growth kinetics and resulting cluster size distributions evolve from the irreversible limit to systems which come to equilibrium over the simulation time due to fragmentation. Depletion-driven colloids, thus provide us with a unique opportunity to study dynamical scaling of time dependent structure factors in a colloidal system which produces both fractal and compact clusters as a function of the strength of the depletion potential. Such a study might provide important insight into the validity and applicability of dynamical scaling for various cluster morphologies. This is the motivation behind the current work.

In this paper, we carry out a detailed simulation of the evolution of the structure factor in depletion-driven colloidal systems, for both shallow and deep quenches into the twophase region. Our results show that true dynamical scaling is reached in a shallow quench (which produces compact clusters) after an initial transient regime. The scaling of the structure factor is confirmed by studying various length scales and by conclusively showing the existence of a single length scale in the system. In contrast, the apparent structure factor scaling for a deep quench (which produces long-lived fractal clusters) is found to be only approximate. Two different length scales are found in the system which grow with two different power-law exponents with time. We further study the origin of the peak in the structure factor in this case and compare with theoretical predictions of HOS by taking cluster polydispersity into account.

\section{NUMERICAL MODEL}

We consider a two-dimensional (2D) system of linear size $L=256 \sigma$ containing $N_{m}=13107$ colloidal particles of diameter $\sigma$. This sets the monomer area fraction to be $f_{v} \approx 0.157$. We also set $\sigma=1$ and thus measure all distances in units of $\sigma$. Periodic boundary conditions are enforced to minimize wall effects. The equations of motion for the colloidal particles in our Brownian dynamics simulations [20], read as

$$
\ddot{\vec{r}}=-\vec{\nabla} U_{i}-\Gamma \overrightarrow{\vec{r}}_{i}+\vec{W}_{i}(t)
$$

where $\Gamma$ is the monomer friction coefficient and $\vec{W}_{i}(t)$, which describes the random force acting on each colloidal particle, is a Gaussian white noise with zero mean and satisfies 2D fluctuation-dissipation relation: $\left\langle\vec{W}_{i}(t) \cdot \vec{W}_{j}\left(t^{\prime}\right)\right\rangle=4 k_{B} T \Gamma \delta_{i j} \delta(t$ $\left.-t^{\prime}\right)$. Hydrodynamic interactions, including lubrication forces, are ignored in the simulation as they might not be of predominant importance for a study of colloids interacting through relatively weak (a few $k_{B} T$ ) attractive potentials [21].

The potential $U$ acting upon each colloidal particle has a twofold contribution: the two-body depletion potential of Asakura-Oosawa [15] $\left(U_{A O}\right)$ plus a repulsive hard-core-like interaction $\left(U_{h c}\right)$ given by the following expressions: 


$$
\frac{U_{A O}\left(r_{i j}\right)}{k_{B} T}=\left\{\begin{array}{ll}
\frac{3 \phi_{P}}{2 \zeta^{3}}\left((1+\zeta)^{2} r_{i j}-\frac{1}{3} r_{i j}^{3}-\frac{2(1+\zeta)^{3}}{3}\right) & \text { for } r_{i j}<(1+\zeta) \\
0 & \text { for } r_{i j}>(1+\zeta)
\end{array}\right)
$$

and

$$
\frac{U_{h c}\left(r_{i j}\right)}{k_{B} T}=r_{i j}^{-n} .
$$

In Eq. (6), $\zeta$ is the size-ratio between a polymer chain and a colloidal particle and is set equal to $\zeta=0.1$ as in previous work $[19,22]$. Thus the interaction is quite short ranged and is cut off at a reduced distance of 1.1. $\phi_{P}$ is the reservoir polymer packing fraction which controls the strength of the depletion interaction in the Asakura-Oosawa model. In the hard-core-like repulsive interaction given by Eq. (7), we have set $n=36$. Exponents $n<36$ are reported [23] to lead to anomalies when a hard-core mimic is required in the potential. The total pair-potential $U=U_{A O}+U_{h c}$ passes through a minimum value $\left(U_{m}\right)$ which is related to $\phi_{P}$. In what follows, we will characterize the strength of the potential in terms of the absolute value of the minimum potential depth, $\left|U_{m}\right|$, instead of $\phi_{P}$.

We choose $\Gamma=0.5$, and a time step $\Delta t=0.005$ in reduced units of $\sigma\left(m / U_{m}\right)^{1 / 2}$ with mass $m=1$. All simulations start from a random homogeneous initial monomer conformation and the results for the kinetics are averaged over 10 runs. The monomer friction coefficient $\Gamma$ is related to the bare diffusion constant $D_{0}$ as $D_{0} \sim k T / \Gamma$. This expression for the diffusion constant would be strictly valid if there were no interactions among the monomers. Nevertheless, this relationship suggests that the actual diffusion constant increases as $\Gamma$ is decreased and this may result in a decrease in crystal nucleation.

\section{RESULTS AND DISCUSSION}

Transition from a single dispersed-phase to a two-phase co-existence is observed in our model when the minimum of the depletion potential, $U_{m}$ is deeper than a critical value $U_{c}$. The monomer area fraction in our simulation is chosen to be $f_{v} \approx 0.157$, and two-phase coexistence is observed when $\left|U_{m}\right|>U_{c} \approx 3.13 k_{B} T$. When $\left|U_{m}\right|$ increases, homogeneous cluster nucleation is observed in the system and, at late times, large round shaped clusters coexist with a sea of monomers and some small aggregates [Fig. 1(a)]. As the inset of Fig. 1(a) shows, colloidal particles are arranged in a hexagonal packing inside such clusters. By increasing $\left|U_{m}\right|$ further, i.e., deep into the two-phase region, fractal clusters are obtained at late times [Fig. 1(b)]. Here, the interfacial tension driven surface reorganization of monomers is almost frozen and the cluster shape results mainly from random cluster-cluster collisions as in a traditional diffusion-limited cluster-cluster aggregation (DLCA) or reaction-limited cluster-cluster aggregation (RLCA) models. However, even for this deep well depth, the aggregates show hexagonal closed-packed crystalline ordering at short length scales [see the inset of Fig. 1(b)] while displaying ramified fractal nature at larger length scales. In the present work, $\left|U_{m}\right|=3.5$ (corresponding to $\phi_{p}=0.241$ ) (shallow quench) and $\left|U_{m}\right|=6.0$ (corresponding to $\phi_{p}=0.333$ ) (deep quench) are selected as representative examples for the study of structure factor scaling in systems displaying compact and fractal clusters, respectively. The phase diagram for the model considered here is known accurately in three dimensions [24,25], but not in two dimensions. For this reason, a quantitative identification of the quench points chosen in our work on the $2 \mathrm{D}$ phase diagram is not possible.

\section{A. General features of the structure factor}

The structure factor $S(\vec{q}, t)$ for a system of $N_{m}$ monomer particles is defined as
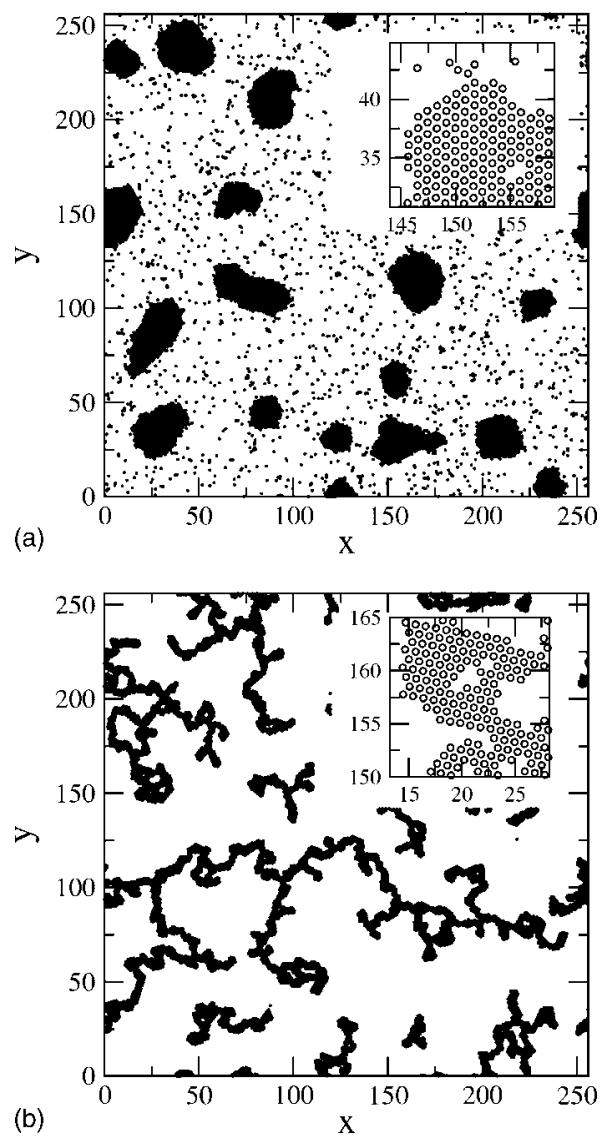

FIG. 1. Cluster morphology at $t=10000$ for (a) shallow $\left(\left|U_{m}\right|\right.$ $=3.5)$ and (b) deep $\left(\left|U_{m}\right|=6.0\right)$ quenches into the two-phase gassolid region. Insets show hexagonal crystalline packing of the colloids inside the clusters. 

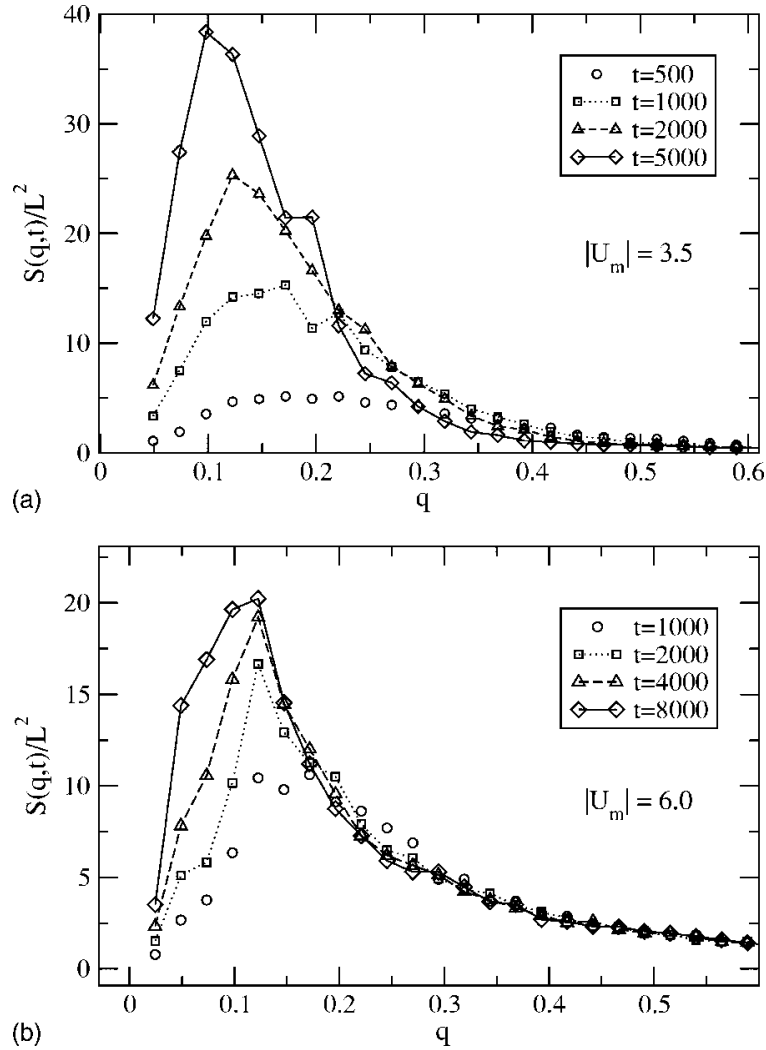

FIG. 2. Structure factors at several times for (a) shallow $\left|U_{m}\right|$ $=3.5$ and (b) deep quenched systems $\left|U_{m}\right|=6$.

$$
S(\vec{q}, t)=\sum_{i}^{N_{m}} \sum_{j}^{N_{m}} \exp \left[i \vec{q} \cdot\left(\vec{r}_{i}-\vec{r}_{j}\right)\right]
$$

The pair-correlation function $g(\vec{r}, t)$ can be obtained from the inverse Fourier transform of $S(\vec{q}, t)$. In order to compute the above quantities we have discretized the system into a $L$ $\times L$ grid of lattice points. We have then calculated the circularly averaged quantities $S(q, t)$ and $g(r, t)$ by using standard fast Fourier transform (FFT) routines.

Figures 2(a) and 2(b) show time-dependent structure factors for shallow $\left(\left|U_{m}\right|=3.5\right)$ and deep quenches $\left(\left|U_{m}\right|=6.0\right)$, respectively. The computed structure factors have been normalized by a factor $L^{2}$, where $L=256$ is the linear size of system. In each case, we observe a peak in the structure factor. The position of the peak moves to smaller $q$-values as time progresses, and the intensity of the peak increases. In contrast, at short length scales (large $q$-values), the structure factor does not evolve much at late times. For this reason, the scaling behavior of the structure factors must be examined carefully over appropriate $q$-values.

For the case of $\left|U_{m}\right|=6.0$, the overlapping of $S(q)$ data for large $q$-values at different times is clearly noticeable. This almost frozen structure at short length scales is directly related to the way in which clusters aggregate after the initial transient time [19]. After an initial period, the small clusters present in the system do not go through any large change of shape or internal rearrangement due to the strong interaction potential. Then, clusters start to collide among themselves
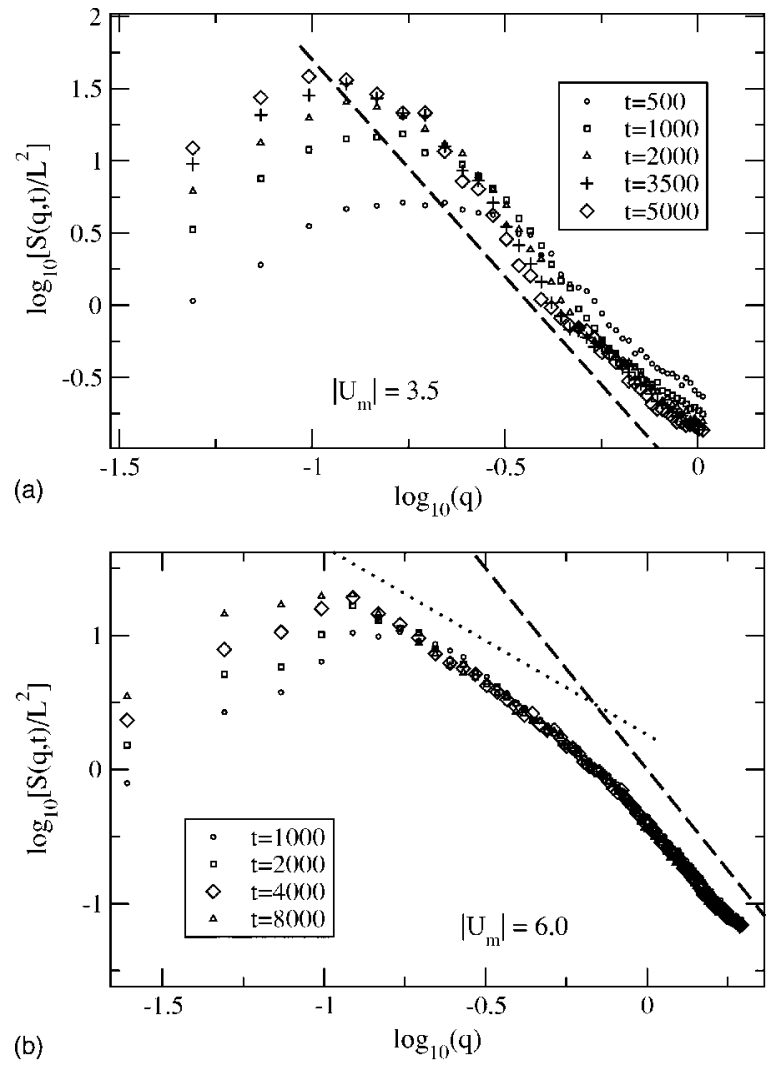

FIG. 3. Log-log plot of the structure factors at several times for $\left|U_{m}\right|=3.5$ and $\left|U_{m}\right|=6.0$. Dashed and dotted lines have been added to guide the eye in the different regimes at large $q$-values. Dashed lines account for the Porod regime $S(q) \sim q^{-(d+1)}(d=2)$, and the dotted line accounts for the intermediate regime predicted for

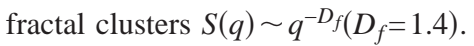

and stick in a way similar to the DLCA model without much internal rearrangements. Therefore the main changes in the system should occur at length scales larger than the former cluster size at that time. As the clusters aggregate and increase their size, the overlap of $S(q, t)$ is expected to start at smaller $q$-values.

Figure 3 shows log-log plots of the structure factors. Porod regime of $S(q) \sim q^{-(d+1)}$ is observed in the case of $\left|U_{m}\right|$ $=3.5$ for large $q$-values. For deep quenched systems, $\left|U_{m}\right|$ $=6$, the fractal nature of the clusters (with $D_{f} \approx 1.4$ ) exhibit a power law regime at intermediate $q$-values, $S(q) \sim q^{-D_{f}}$. With $\left|U_{m}\right|=6$, we also observe a Porod's regime for larger $q$ values, originating from the short-range crystalline packing of the monomers.

\section{B. Scaling of the structure factor and various length scales in the system}

\section{Growth of compact clusters}

The time evolution of the structure factor can be characterized by using the standard scaling ansatz, Eq. (2). Several quantities can be proposed as representative characteristic length scales of the system: the average cluster radius of gyration $R_{g}(t)$, the cluster-cluster nearest-neighbor distance 
$R_{n n}(t)$, the first zero $R_{0}(t)$ of the connected part of the paircorrelation function (a measure commonly used in numerical studies of phase separation in binary mixtures [26]), and the inverse of the location of the peak of the structure factor, $q_{m}^{-1}(t)$. In order to distinguish the growing clusters from the background sea of monomers and small clusters, our calculations of $R_{n n}(t)$ and $R_{g}(t)$ are carried out considering only clusters of size $>4$.

Figures 4(a)-4(c) show scaling plots for time-dependent structure factors for shallow quenched systems $\left(\left|U_{m}\right|=3.5\right)$ using $R_{g}, R_{0}$ and $\left(q_{m}\right)^{-1}$ as characteristic length scales, respectively. The three scaling plots show the same trend: after an initial transient period, $t_{i} \sim 10^{3}-2 \times 10^{3}$, the data scale nicely onto a master curve. As shown on Fig. 4(c), the shape of the scaling function agrees quite well with the form predicted by Furukawa [27] for shallow quenches.

Since scaling seems to work well at late times with various representations of the characteristic length scale, one might conclude that a single effective length scale must exist in the system for the growth of compact clusters. Still a detailed look at the evolution of various length scales is important for a stringent test of the scaling behavior. We must add that we do not expect any deviation from scaling for the evolution of compact clusters at late times, but characterization of the various length scales for this relatively simpler system will provide insight into a more complicated case analyzed later.

In order to make sure of the existence of a single, characteristic length scale in the system, we have plotted in Fig. 5 the temporal evolution of several ratios of various length scales mentioned previously. The behavior of these various ratios is similar in all cases: after the initial transient period $t_{i}$, all the length scales in the system evolve in a similar way, and are proportional to each other. In addition, Fig. 6 shows individual temporal evolution of $R_{n n}(t)$ and $R_{g}(t)$ in a log-log plot where only clusters of size $>4$ are considered. At late times, both quantities are observed to evolve with the same power law exponent [see Eq. (4)], with $n_{1}=n_{2} \approx 0.25$. A power-law exponent of $\frac{1}{4}$ indicates that cluster growth at these times is dominated by collisions among large clusters and a corresponding reduction in the interfacial energy by surface diffusion [1].

Now we would like to understand the origin of the breakdown of scaling at earlier times for compact cluster growth. To understand this we need to look at the temporal evolution of $R_{g}$ and $R_{n n}$ carefully. From Fig. 6, we note that at very early times, $t<t_{1} \approx 300, R_{g}$ grows very slowly. In the next stage, $t>t_{1}$, cluster growth is very fast. This enhanced growth rate lasts up to a second characteristic time $t_{2}$ $\approx 1000$ after which a power-law growth of $R_{g}$ with an exponent $n_{1} \simeq 0.25$ is observed. Similarly, three different regimes seem to exist for the growth of the nearest-neighbor cluster distance, $R_{n n}$. For $t<t_{1}, R_{n n}$ actually decreases before reaching a plateau. For $t_{1}<t<t_{2}, R_{n n}$ grows very fast and then, beyond $t_{2}$, a power-law growth of $R_{n n}$ with an exponent $n_{2}$ $\simeq 0.25$ is observed. It is in this later time regime, $t>t_{2}$ (i.e., the initial transient time $t_{i} \approx t_{2}$ ) that the system shows scaling behavior with a single, characteristic length scale.

The three regimes observed in Fig. 6 can be explained as follows. During the first stage $t<t_{1}$, cluster nuclei are
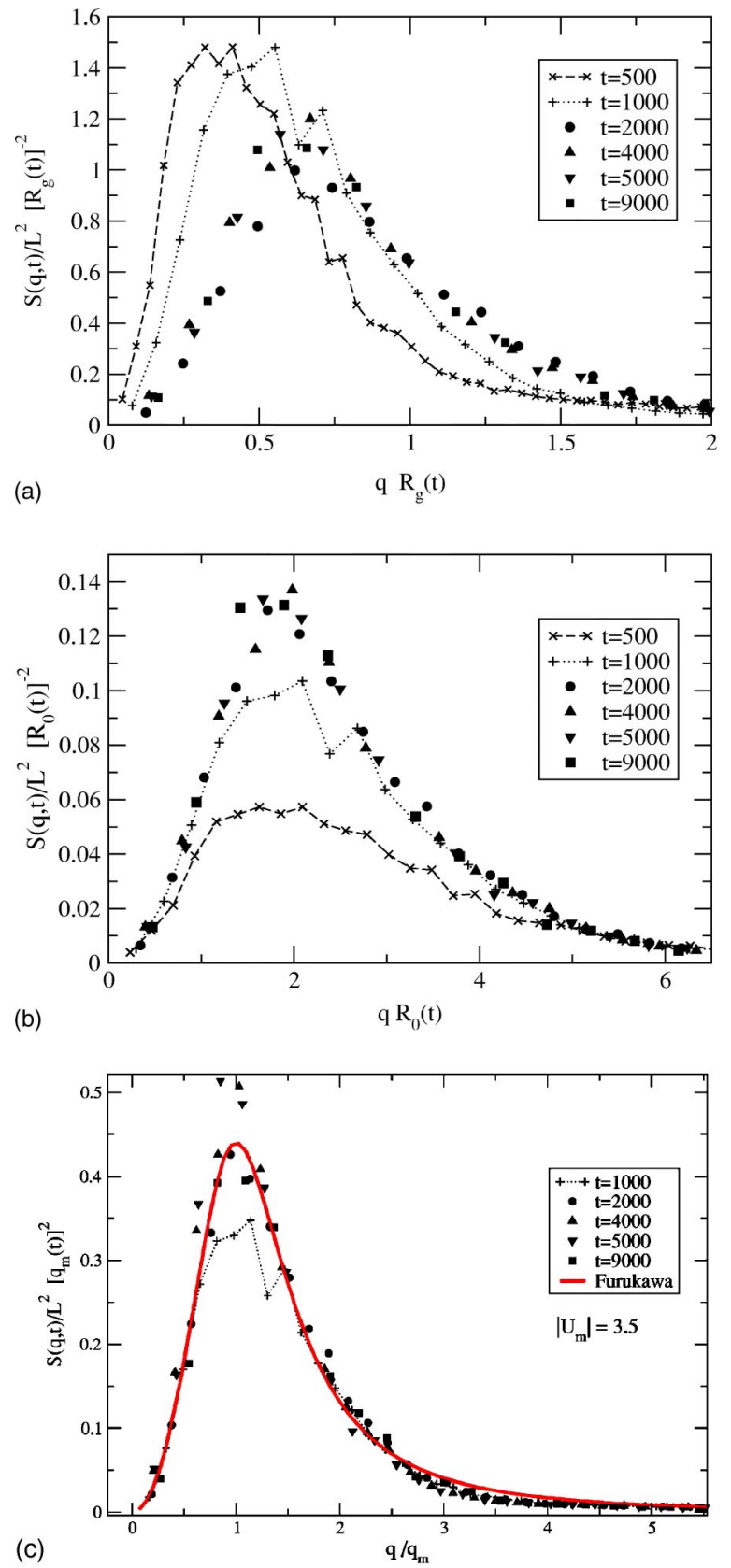

FIG. 4. Scaling plots of the structure factor for $\left|U_{m}\right|=3.5$. Characteristic lengths used are (a) the mean cluster gyration radius $R_{g}$, (b) the first zero of the connected part of the correlation function $R_{0}$, and (c) the inverse of the peak position $q_{m}$ of the structure factor. Filled symbols are used for times at which scaling holds. In (c) the solid line is the scaling form predicted by Furukawa [27] for shallow quenches.

formed, and during this initiation period, the mean radius of gyration of clusters remains approximately constant. This value of $R_{g}$ is expected to be close to the mean critical nucleation radius. During this period, the formation of nuclei leads to a decrease of the mean distance between clusters. This is clearly demonstrated in the observed decrease of $R_{n n}$ at this early stage. Thus, the first regime $t<t_{1}$ can be identified as a nucleation period in which nucleation of small aggregates takes place. Given the different behavior of $R_{g}$ and $R_{n n}$ in this 


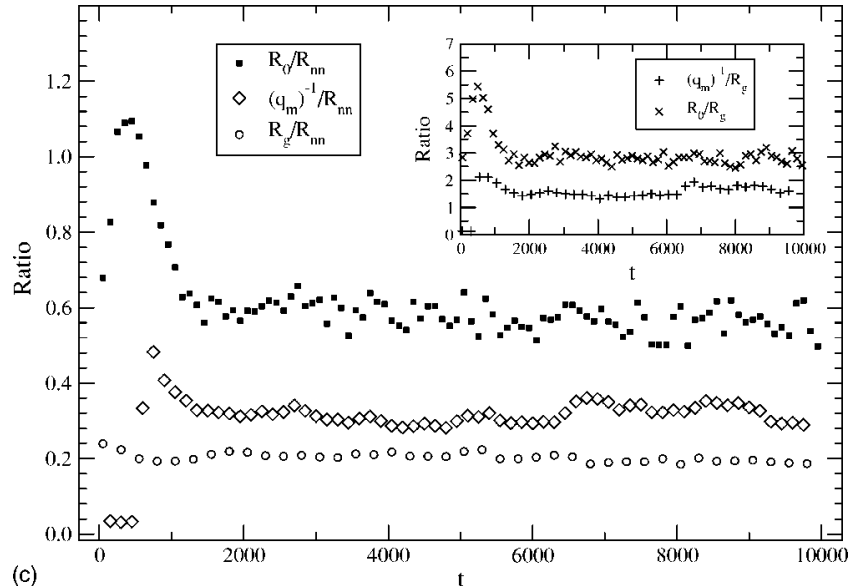

FIG. 5. A comparison of the temporal evolution of various ratios of possible characteristic lengths: $R_{g}, R_{n n}, R_{0}$, and $\left(q_{m}\right)^{-1}$ for $\left|U_{m}\right|$ $=3.5$.

first stage, no scaling with a single length scale is possible.

By plotting the total mass of the small clusters (of size $<5$ ) versus time, we find that the characteristic time $t_{2}$ of the second stage coincides with the time when the total mass of the population of single particles and small aggregates (i.e., clusters containing less than 5 particles) reaches a steady state. This simulation result suggests that beyond this time $t_{2}$, the net flux of small aggregates becomes zero, i.e., the number of small aggregates entering large clusters equals the number of small aggregates leaving large clusters. Thus, for $t>t_{2}$, the predominant mechanism of cluster growth is due to collision among large clusters and a subsequent reduction of the total interfacial energy as surface area minimizes. On the other hand, at least two different mechanisms contribute to the cluster growth in the intermediate regime $t_{1}<t<t_{2}$. It is easy to show that the mechanism of collision among large clusters leads to the same growth law exponent for $R_{g}$ and $R_{n n}$ if the clusters are assumed to be compact: the free space that surrounds the new cluster after the collision grows lin-

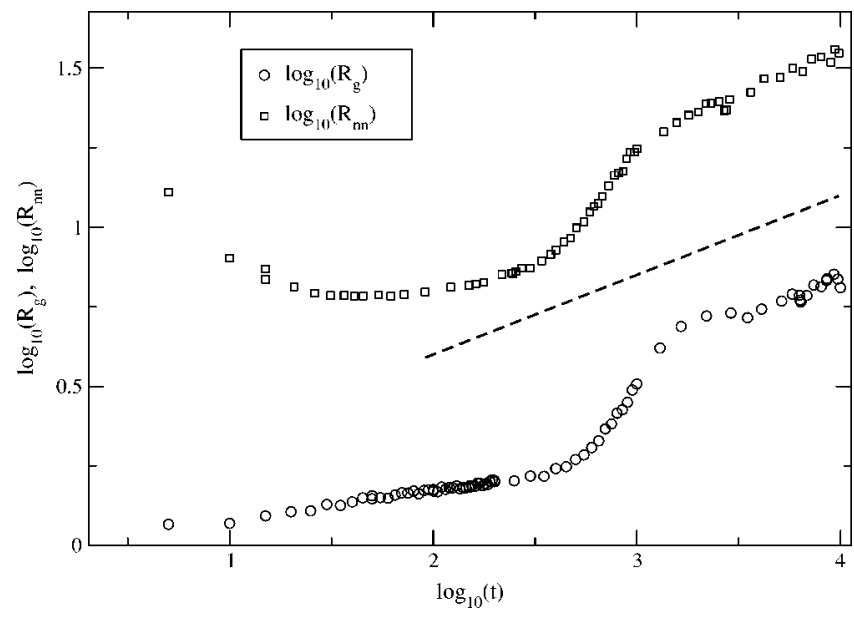

FIG. 6. The temporal evolution of $R_{g}$ and $R_{n n}$ for $\left|U_{m}\right|=3.5$. After an initial transient period $t_{i} \sim 10^{3}-2 \times 10^{3}$, both length measures evolve closely in time as $\sim t^{0.25}$. A line of slope 0.25 has been included to guide the eye. early with the new size of the cluster. In contrast, a growth mechanism based on a net flux of single particles and small aggregates towards the large clusters leads to a different temporal evolution of $R_{n n}$ and $R_{g}$. This is due to the fact that the distance between the center of masses of the large clusters remains constant on average, but the radius of gyration grows due to the incorporation of new particles. Therefore, when both growth mechanisms are present in the system as in the intermediate stage $t_{1}<t<t_{2}$, dynamical scaling cannot be expected, even for compact clusters, as $R_{n n}$ and $R_{g}$ would evolve differently in time. Only beyond a characteristic time $t_{2}$, when the the net flux of small aggregates becomes zero, the evolution of $R_{n n}$ and $R_{g}$ becomes proportional to each other and dynamical scaling of the structure factor holds.

\section{Growth of fractal clusters}

The scaling behavior of the structure factor for a deep quench into the two-phase region, $\left|U_{m}\right|=6.0$, is showed in Figs. 7(a)-7(c) with $R_{g}, R_{0}$, and $q_{m}^{-1}$ being used as measures of the characteristic length scale of the system, respectively. For fractal aggregates, the spatial dimension $d$ in the scaling form [Eq. (2)] is replaced by the fractal dimension $D_{f}$; such a form has been expressed in Eq. (3). We note that a scaling description is meaningful only for intermediate values of $q$ since at small length scales (large $q$-values) an almost frozen hexagonally-packed crystal structure is observed and the structure factor does not evolve much for large $q$-values at late times [see Fig. 2(b)]. At late times, data for intermediate values of $q$ seems to fall on a master curve indicating a dynamical scaling behavior. Scaling seems to be particularly good when $R_{0}$, and $q_{m}^{-1}$ are used as characteristic length scales. Such a scaling behavior was observed previously in 2D aggregation of polystyrene colloids [8] and in numerical simulations of the DLCA model [28]. However, as we present shortly, a detailed study of the various length scales in the system suggests that this apparent scaling of the structure factor is not a signature of dynamical scaling in a strict sense.

In Fig. 8 we show the evolution of $R_{g}$ and $R_{n n}$ versus time $t$ in a log-log plot. It is clear that these two length scales evolve differently in time with growth exponents $n_{1}$ $=0.52 \pm 0.03$ and $n_{2}=0.36 \pm 0.03$, defined in Eq. (4). How does one understand these values of the growth exponents?

As mentioned before and illustrated in Ref. [19], the interfacial tension driven surface reorganization of monomers is almost frozen for deep quenches, and the cluster shape results mainly from random cluster-cluster collisions as in a traditional DLCA model. Then, scaling arguments of Refs. $[29,30]$ for the DLCA model should be applicable here. For DLCA model with a Brownian coagulation kernel one finds that the homogeneity constant, $\lambda$, of the aggregation kernel is given by $\lambda=(d-3) / D_{f}$ in the dilute limit. The kinetic exponent $z$, which describes how the mean cluster size $s(t)$ scales with time $t$, is in turn related to $\lambda: z=1 /(1-\lambda)$. In $3 \mathrm{D}$, this provides $\lambda=0$ and $z=1$ as expected [31]. In 2D, however, this leads to $\lambda=-1 / D_{f}=-0.7$ with $D_{f}=1.4$ and hence $z$ $=0.59$ in the dilute limit. However, it is known that the kinetic exponent $z$ increases as the system gets dense [29], i.e., as the volume fraction occupied by the clusters, $f_{v}^{c}$, increases. 

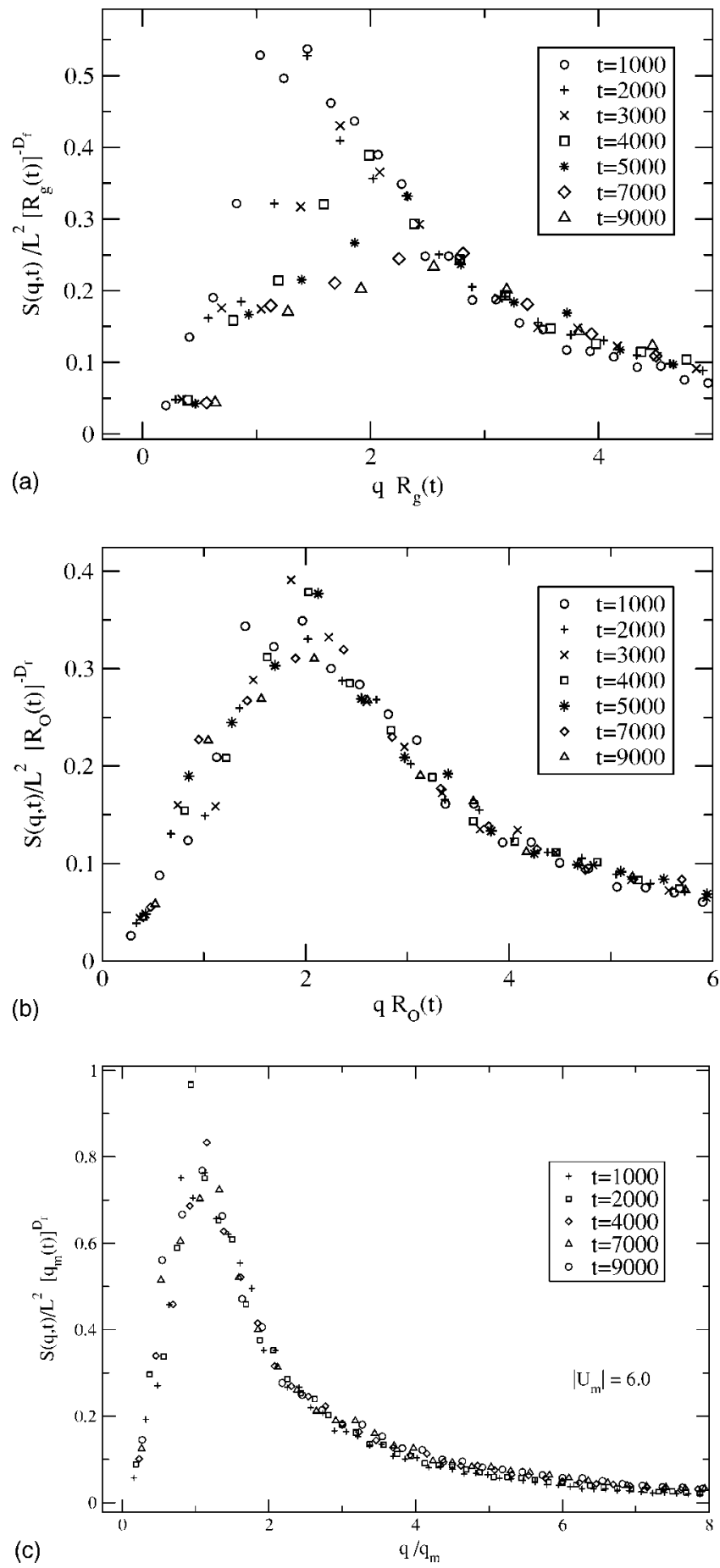

FIG. 7. Scaling plots of the structure factor for $\left|U_{m}\right|=6.0$. Characteristic lengths used are (a) the mean cluster gyration radius $R_{g}$, (b) the first zero of the connected part of the correlation function $R_{0}$, and (c) the inverse of the peak position $q_{m}$ of the structure factor. Fractal dimension is set to $D_{f}=1.4$.

For an intermediate value of $f_{v}^{c}\left(0.1<f_{v}^{c}<1\right)$, scaling arguments yield $z \simeq 1.28$ in $3 \mathrm{D}$ and $z \simeq 0.67$ in $2 \mathrm{D}$. Simulations of the AO model [19] yield a $z$-value rather similar to the $2 \mathrm{D}$ scaling result in the intermediate regime mentioned above and also to the kinetic exponent obtained in large-scale 2D

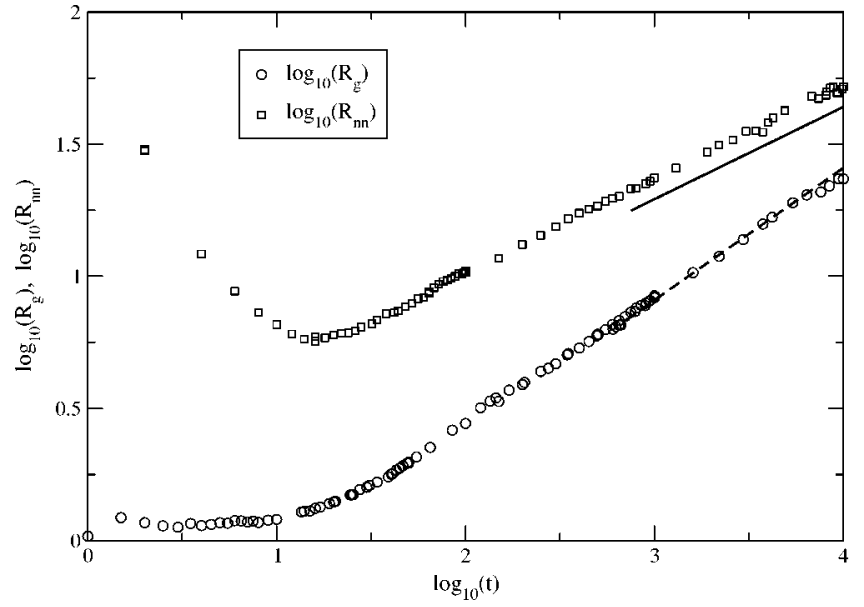

FIG. 8. The temporal evolution of $R_{g}$ and $R_{n n}$ for $\left|U_{m}\right|=6.0$. For deep quenched systems, the evolution of $R_{g}$ and $R_{n n}$ is different with time. A solid line of slope 0.36 and a dashed line of slope 0.52 have been included to guide the eye.

DLCA simulations with a fair degree of cluster crowdedness [32].

The kinetic exponent $z$ is related to the growth exponent $n_{1}$ for the temporal evolution of the mean cluster radius of gyration, $R_{g}$. In the scaling description of DLCA, $n_{1}=z / D_{f}$. Following Ref. [19] if we consider $z=0.74$ and $D_{f}=1.4$ in $2 \mathrm{D}$, we obtain $n_{1}=0.53$ in excellent agreement with the observation of Fig. 8. On the other hand, $R_{n n}$ scales as $N_{c}^{-1 / d}$ where $N_{c}$ is the number of clusters at time $t$. Since the total number of monomers is constant in the system, one can write $R_{n n} \sim s^{1 / d}$ where $s$ is the mean cluster size at time $t$. As $s$ $\sim t^{z}$, one obtains $R_{n n} \sim t^{z / d}$. Thus, $n_{2}=z / d=0.37$ in $2 \mathrm{D}$, again in good agreement with the value obtained in Fig. 8.

Since $n_{1} \neq n_{2}$, it is clear that a single characteristic length does not exist in the system. This is a consequence of the fractal nature of the clusters [11]. The breakdown of scaling is further demonstrated in Fig. 9 by plotting ratios of various length scales in the system. As expected, $R_{g} / R_{n n}$ is not a

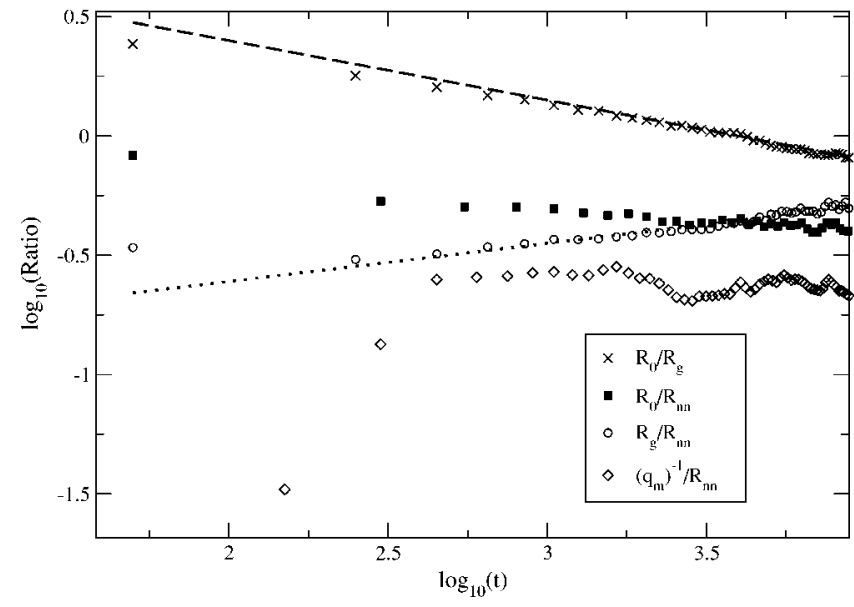

FIG. 9. A comparison of the temporal evolution of various ratios of possible characteristic lengths: $R_{g}, R_{n n}, R_{0}$, and $\left(q_{m}\right)^{-1}$ for $\left|U_{m}\right|$ $=6$. Dashed and dotted lines are guides to the eye with slopes -0.25 and 0.16 , respectively. 


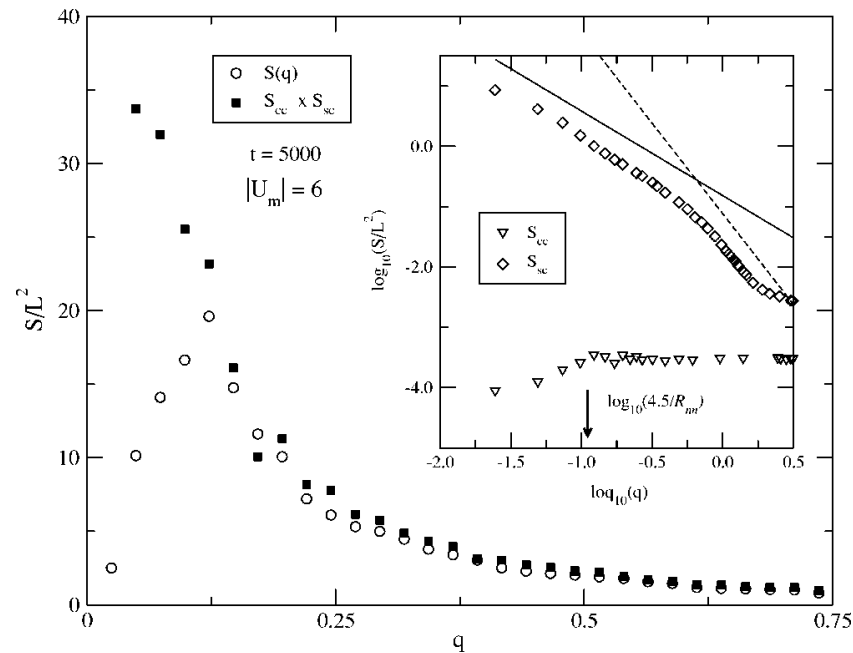

FIG. 10. The structure factor obtained for $\left|U_{m}\right|=6.0$ at time $t$ $=5000$ is compared with HOS prediction $S=S_{c c} \times S_{s c}$. The inset depicts the behavior of $S_{c c}$ and $S_{s c}$ separately in log-log plots. In the inset two lines are plotted to guide the eye for $S_{s c}$ : dashed line stands for a slope equal to $-(d+1)=-3$, and solid line stands for a slope equal to $-D_{f}=-1.4$. $S_{c c}$ flattens out at a $q$ value around $4.5 R_{n n}^{-1}$.

constant in time but rather grows with an exponent given by $n_{1}-n_{2}=0.16$. Similarly, the ratio $R_{0} / R_{g}$ shows clear time dependence. It is interesting to note that both $q_{m}^{-1} / R_{n n}$ and $R_{0} / R_{n n}$ show weak dependence on $t$. This indicates that, both $q_{m}^{-1}$ and $R_{0}$ are closely related to one of the characteristic length scale of the system, namely, $R_{n n}$. We will address this point further in the next section.

Now that we clearly demonstrate the existence of more than one length scale in the system, why does the structure factor shows scaling as illustrated in Fig. 7? Note that, as shown in Fig. 8, the two length scales $R_{g}$ and $R_{n n}$ approach each other as time progresses. Since their ratio $R_{n n} / R_{g}$ grows weakly with time $t$, the relative difference between these two length scales tend to decrease as time increases. This opens up a window in time where these two length scales are of similar magnitude, and scaling over this limited time interval seems to satisfy. Dynamical scaling as a general principle fails though for the growth of fractal clusters.

\section{Origin of the peak in the structure factor}

HOS has explained the presence of a peak in the structure factor by writing the total structure factor for monodisperse systems as a combination of two structure factors: the singlecluster structure factor $S_{s c}$, which involves the cluster radius of gyration $R_{g}$ as the characteristic length, and the clustercluster structure factor $S_{c c}$, which involves the mean nearestneighbor distance $R_{n n}$ as the characteristic length. In moderately dense systems, this product causes a peak in the structure factor but the peak location does not represent a true length scale of the system. $q_{m}^{-1}$ is thus not an independent characteristic length scale, but rather can be expressed as a linear combination of $R_{g}$ and $R_{n n}$.

Figure 10 shows the comparison of the total structure fac-

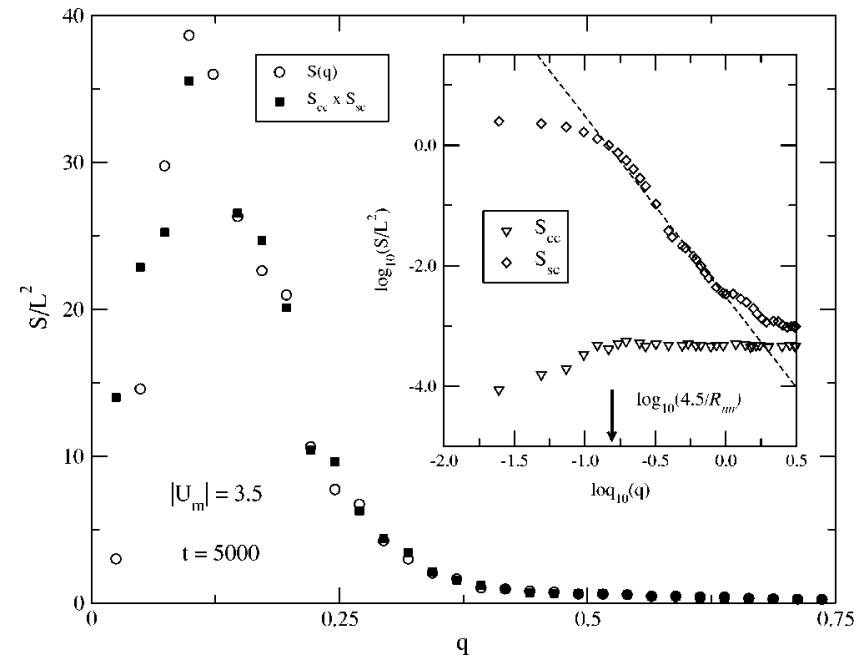

FIG. 11. The structure factor obtained for $\left|U_{m}\right|=3.5$ at time $t$ $=5000$ is compared with HOS prediction $S=S_{c c} \times S_{s c}$ where only clusters larger than $N>30$ have been taken into account for the calculation of $S_{c c}$ and $S_{s c}$. The inset depicts the behavior of $S_{c c}$ and $S_{s c}$ separately. A dashed line with a slope equal to $-(d+1)=-3$ is shown to guide the eye. $S_{c c}$ flattens out at a $q$ value around $4.5 R_{n n}^{-1}$.

tor with the product $S_{c c} \times S_{s c}$ for deep quenches at $t=5000$. The inset of Fig. 10 shows $S_{c c}$ and $S_{s c}$ separately in a log-log plot. The cluster-cluster structure factor $S_{c c}$, has been computed by considering the centers of mass of the clusters as scattering points. The single cluster structure factor $S_{s c}$ has been obtained by computing the individual cluster structure factors and averaging over all clusters. As it is shown in Fig. 10 , the product $S_{c c} \times S_{s c}$ is a reasonably good approximation for the total structure factor for large $q$-values. The inset of Fig. 10 shows the two power-law regimes for $S_{s c}$ : a regime in which $S_{s c}(q) \sim q^{-D_{f}}$ and at larger $q$-values, a regime in which $S_{s c}(q) \sim q^{-(d+1)}$ according to Porod's law, originating from the short-range crystalline order in the aggregates. $S_{c c}$ on the other hand, flattens out at a $q$ value around $4.5 R_{n n}^{-1}$, in good agreement with HOS.

Unfortunately, the measured value of $S_{c c} \times S_{s c}$ does not reproduce the observed peak in the total structure factor from the simulation. Instead, this product shows a monotonically decreasing function. One possible reason for this disagreement at small $q$-values is the polydispersity of the cluster size distribution for deep quenches. In shallow quenched systems $\left(\left|U_{m}\right|=3.5\right)$, however, one finds large clusters with a relative small degree of polydispersity if we do not take into account the very small aggregates and single colloidal particles that surround the large clusters. Therefore, $\left|U_{m}\right|=3.5$ quenches seem to be a more suitable system for testing HOS predictions applicable for monodisperse systems. Figure 11 shows a comparison of the total structure factor for $\left|U_{m}\right|=3.5$ at $t$ $=5000$ with the product $S_{c c} \times S_{s c}$ where only clusters larger than $N>30$ are taken into account. The inset of Fig. 11 shows the behavior of $S_{c c}$ and $S_{s c}$ in this case. The dashed line shows the predicted Porod regime for $S_{s c}$ in the case of compact clusters: $S_{s c}(q) \sim q^{-(d+1)}$. $S_{c c}$ again flattens out at a $q$ value around $4.5 R_{n n}^{-1}$, in good agreement with HOS. In this case, we observe that the product $S_{c c} \times S_{s c}$ reproduces the 
peak of the total structure factor. Thus it quite possible that the absence of the peak in the product $S_{c c} \times S_{s c}$ for $\left|U_{m}\right|=6$ is due to the polydispersity in the cluster size.

In our simulations for both shallow and deep quenches, the peak position $q_{m}^{-1}$ is found to be closely related to the nearest neighbor cluster-cluster separation $R_{n n}$. For compact cluster growth, there is only one length scale in the system and thus $q_{m}^{-1}$ can also be expressed in terms of the mean radius of gyration of the growing clusters. For fractal cluster growth, there are two independent length scales but over the simulation time, $q_{m}^{-1}$ is found to be proportional to $R_{n n}$.

\section{CONCLUDING REMARKS}

An important result of this work is the demonstration that aggregating colloids exhibit a true structure factor scaling only when the growing clusters are compact. This can be achieved for shallow quenches into the two-phase region of a depletion-driven colloid. For such quenches, true scaling occurs after an initial transient time. This transient period covers nucleation of clusters and growth mainly by incorporating monomers and small clusters in the growing nuclei. In this regime, $R_{n n}$ and $R_{g}$ grow differently with time and scaling does not work. After the transient period, the predominant mechanism of cluster growth is collision among large clusters and the subsequent surface reorganization of clusters to reduce interfacial tension. This process leads to a similar temporal evolution of $R_{n n}$ and $R_{g}$, and a single length scale can be observed in the system. Dynamical scaling is thus satisfied.
For deep quenches, the magnitude of the interaction potential between colloidal particles is much larger than thermal energy $k_{B} T$. Thus, both rearrangement and fragmentation of clusters are practically frozen and the growing clusters are fractals over long periods of time. Simulation results show that cluster growth in this regime is controlled by two characteristic lengths $R_{n n}$ and $R_{g}$ that evolve differently with time. True dynamical scaling is thus not possible although an apparent scaling of the structure factor is observed when these two length scales are comparable in magnitude.

Another important result of this work is to understand the shape of the total structure factor. In this respect, our results are compared with the predictions of HOS. HOS predict that the total structure factor for a monodisperse system can be described as a product of the cluster-cluster and the averaged-single-cluster structure factors, each with its own characteristic length. In the HOS formulation, the peak in the total structure factor then arises due to the overlap of these two contributions. Simulation results show that this description works for monodisperse systems but seems to break down for polydisperse cluster size distributions.

Much of the simulation results presented here are amenable to direct experimental tests. We hope that our work would stimulate further experimental and theoretical studies towards the full understanding of the aggregation processes in widely different physical situations.

\section{ACKNOWLEDGMENTS}

J.J.C. and T.S. acknowledge financial support from the Spanish MCyT Grant No. BMF2001-0341-C02-01. A.C. and C.S. are supported by NASA Grant No. NNC04GA74G.
[1] J. D. Gunton, M. san Miguel, and P. S. Sahni, in Phase Transition and Critical Phenomena, edited by C. Domb and J. L. Lebowitz (Academic, New York, 1983), Vol. 8.

[2] A. J. Bray, Adv. Phys. 43, 357 (1994).

[3] K. Binder and P. Fratzl, in Phase Transformations in Materials, edited by G. Kostorz (Wiley-VCH, Weinheim, 2001).

[4] See, for example, K. Binder in Materials Science and Technology, Vol. 5: Phase Transformations in Materials, edited by P. Haasen (Wiley-VCH, Weinheim, 1990).

[5] A. Chakrabarti, Phys. Rev. B 45, 9620 (1992).

[6] M. Carpineti and M. Giglio, Phys. Rev. Lett. 68, 3327 (1992); 70, 3828 (1993).

[7] J. Bibette, T. G. Mason, H. Gang, and D. A. Weitz, Phys. Rev. Lett. 69, 981 (1992).

[8] D. J. Robinson and J. C. Earnshaw, Phys. Rev. Lett. 71, 715 (1993).

[9] J. C. Earnshaw, M. B. J. Harrison, and D. J. Robinson, Phys. Rev. E 53, 6155 (1996).

[10] Kinetics of Aggregation and Gelation, edited by F. Family and D. Landau (North-Holland, Amsterdam, 1984).

[11] H. Huang, C. Oh, and C. M. Sorensen, Phys. Rev. E 57, 875 (1998)

[12] For a review, see W. C. K. Poon and M. D. Haw, Adv. Colloid Interface Sci. 73, 71 (1997).

[13] For a review, see V. J. Anderson and H. N. W. Lekkerkerker,
Nature (London) 416, 811 (2002).

[14] J. Israelachvili, Intermolecular and Surface Forces (Academic, San Diego, 1994).

[15] S. Asakura and F. Oosawa, J. Chem. Phys. 22, 1255 (1954).

[16] A. Vrij, Pure Appl. Chem. 48, 471 (1976).

[17] R. Verma, J. C. Crocker, T. C. Lubensky, and A. G. Yodh, Macromolecules 33, 177 (2000).

[18] E. K. Hobbie, Phys. Rev. Lett. 81, 3996 (1998).

[19] Juan J. Cerda, T. Sintes, C. M. Sorensen, and A. Chakrabarti, Phys. Rev. E 70, 011405 (2004).

[20] W. F. van Gunsteren and H. J. C. Berendsen, Mol. Phys. 45, 637 (1982).

[21] N. Q. Nguyen and A. J. C. Ladd, Phys. Rev. E 66, 046708 (2002); J. F. Brady, J. Chem. Phys. 99, 567 (1993); D. O. Riese et al., Phys. Rev. Lett. 85, 5460 (2000).

[22] K. G. Soga, J. R. Melrose, and R. C. Ball, J. Chem. Phys. 110, 2280 (1999).

[23] J. R. Melrose, Europhys. Lett. 19, 51 (1992).

[24] H. N. W. Lekkerkerker, W. C. K. Poon, P. N. Pusey, A. Stroobants, and P. B. Warren, Europhys. Lett. 20, 559 (1992).

[25] M. Dijkstra, J. M. Brader, and R. Evans, J. Phys.: Condens. Matter 11, 10079 (1999).

[26] D. A. Huse, Phys. Rev. B 34, 7845 (1986).

[27] H. Furukawa, Phys. Rev. B 33, 638 (1986); Physica A 123, 497 (1984). 
[28] T. Sintes, R. Toral, and A. Chakrabarti, Phys. Rev. E 50, R3330 (1994).

[29] D. Fry, T. Sintes, A. Chakrabarti, and C. M. Sorensen, Phys. Rev. Lett. 89, 148301 (2002).

[30] A. Chakrabarti, D. Fry, and C. M. Sorensen, Phys. Rev. E 69,
031408 (2004).

[31] S. K. Friedlander, Smoke, Dust and Haze (Oxford, New York, 2000).

[32] D. Fry, Ph.D. thesis, Kansas State University, 2003; D. Fry, F. Pierce, C. M. Sorensen, and A. Chakrabarti (unpublished). 